\title{
Analysis of numerical studies on the thermal-hydraulic and neutronic thermal-hydraulic stability of supercritical water reactors
}

\author{
Artavazd M. Sujyan ${ }^{1}$, Viktor I. Deev ${ }^{1}$, Vladimir S. Kharitonov ${ }^{1}$ \\ 1 National Research Nuclear University MEPhI, 31 Kashirskoe Shosse, Moscow, 115409, Russia \\ Corresponding author: Artavazd M. Sujyan (artavazd1994@gmail.com)
}

Academic editor: Georgy Tikhomirov • Received 16 June 2021 Accepted 24 October 2021 • Published 17 December 2021

Citation: Sujyan AM, Deev VI, Kharitonov VS (2021) Analysis of numerical studies on the thermal-hydraulic and neutronic thermalhydraulic stability of supercritical water reactors. Nuclear Energy and Technology 7(4): 311-318. https://doi.org/10.3897/nucet.7.78368

\begin{abstract}
The paper presents a review of modern studies on the potential types of coolant flow instabilities in the supercritical water reactor core. These instabilities have a negative impact on the operational safety of nuclear power plants. Despite the impressive number of computational works devoted to this topic, there still remain unresolved problems. The main disadvantages of the models are associated with the use of one simulated channel instead of a system of two or more parallel channels, the lack consideration for neutronic feedbacks, and the problem of choosing the design ratios for the heat transfer coefficient and hydraulic resistance coefficient under conditions of supercritical water flow. For this reason, it was decided to conduct an analysis that will make it possible to highlight the indicated problems and, on their basis, to formulate general requirements for a model of a nuclear reactor with a light-water supercritical pressure coolant. Consideration is also given to the features of the coolant flow stability in the supercritical water reactor core. In conclusion, the authors note the importance of further computational work using complex models of neutronic thermal-hydraulic stability built on the basis of modern achievements in the field of neutron physics and thermal physics.
\end{abstract}

\section{Keywords}

Nuclear reactor, supercritical water, coolant flow instabilities, computational models

\section{Introduction}

An important task in the research of nuclear power plants is to determine the boundaries of their stable operation modes, which is necessary to ensure safety. In this context, 'instability' means the occurrence of sustained oscillations in the coolant flow rate when small short-term disturbances are introduced into the system. There are two main types of stability related to supercritical pressure water reactors
(SCWR): (1) thermal-hydraulic, which is characterized by the retention of operating parameters (density, temperature, pressure and flow rate) of the coolant flow, as well as heat transfer parameters during operation, and (2) neutronic thermal-hydraulic, which consists in maintaining the stability of the processes of energy release and heat removal in the core. The whole set of different types of instability can be divided into two main groups: static and dynamic (oscillatory) instabilities (Bergles 1977, Yadigaroglu 1984).

* Russian text published: Izvestiya vuzov. Yadernaya Energetika (ISSN 0204-3327), 2021, n. 3, pp. $29-43$. 
A typical example of static instability is the Ledinegg instability (Ambrosini 2007), associated with the ambiguity of the hydrodynamic characteristics of the channel, i.e., different values of the coolant flow rate at the same pressure drop. The usual way to eliminate this type of instability is to increase the hydraulic resistance of the inlet section, for example, by installing a choke at the inlet to the channel.

The characteristic types of dynamic instability are thermoacoustic oscillations and oscillations of the 'density wave' type (Bergles 1977). Occurrence of oscillatory processes in a medium of supercritical parameters is sometimes associated with 'pseudo-boiling'. Thus, for example, the processes of compression and expansion of gas moles, represented by pseudo-bubbles, are considered to be the cause of thermoacoustic oscillations arising in a supercritical fluid (Ambrosini 2007). Density wave instabilities deserve the most attention in the development of SCWRs. If feedbacks are available between various parameters of the system, transport and inertial effects lead to a certain delay in the passage of disturbances along the channel length. Thus, temperature (enthalpy) disturbances cause coolant density oscillations, which propagate along the channel length in the form of density waves, causing pressure drop pulsations. Through the feedback, there appear corresponding pressure and temperature disturbances. With a certain phase correspondence, the oscillations in the operating parameters become self-sustaining, which is highly undesirable.

To analyze the dynamic instability of the coolant flow in the core of nuclear reactors, it is necessary to consider complex oscillatory flow regimes in parallel channels. In this case, instabilities of the coolant flow appear when there is a noticeable difference in the specific flow volumes in the parallel channels or in modes with natural circulation with noticeably different coolant heating in the parallel channels. Therefore, in designing reactor plants, it is practically important to establish the boundaries of the region of existence of oscillatory modes as well as their main dynamic characteristics. For this reason, the purpose of the work is to analyze the existing results in the field of computational research of thermal-hydraulic and neutronic thermal-hydraulic stability of supercritical water reactors as well as to specify problems for further research in this area.

\section{Results of thermal-hydraulic stability studies}

A significant number of works have been devoted to the problem of establishing the boundaries of the region of existence of oscillatory modes. Most of them were performed by computational methods using various types of computational models built for heated channels of a simple geometric shape (round pipes) or individual cells that include fuel elements and a coolant. Computational models are basically built by two methods, i.e, frequency and time analyses, which differ in how the initial equations and uniqueness conditions are written.

In the frequency analysis method, the nonlinear differential equations of the model are linearized near the operating point, and a stability map is constructed for the system under study. However, due to this linearization, information about the system nonlinear properties is lost.

To simulate nonlinear processes in the system under study, the time analysis method is used, which involves nonlinear models with partial differential equations in space and time. As a rule, this method is used to study transient processes. At the initial stage, a stationary solution to the problem is obtained, after which a small disturbance is imposed on the stable state to obtain a dynamic response of the system. If the disturbance grows over time and leads to oscillations in the coolant flow rate, the system is considered unstable. If, after the disturbance, the oscillations damp, and the initial stationary state is established, then the system is stable.

As the analysis of the research results shows (Ambrosini 2007, Ambrosini and Sharabi 2008, Ambrosini et al. 2011), the influence of operating and design parameters on the position of the flow stability boundaries for media at supercritical pressures is similar to the influence of the same parameters for a two-phase flow. Due to the fact that at supercritical pressures the dependence of density on enthalpy $\rho(h)$ is less sharp than at a phase transition in the pressure range $p<p_{\mathrm{cr}}$, the flow of a supercritical medium should be more stable than a two-phase one, while the stability region should expand as the pressure increases.

The main parameters influencing the stability of the flow of a supercritical medium in channels of a simple geometric shape include pressure $p$; mass flow rate in the channel $\rho w$; heat flux density on the heat-transfer surface $q$; fluid subcooling at the inlet to the temperature of the pseudophase transition expressed through the difference in enthalpies $\Delta h_{\text {in }}=h_{\text {heat }}-h_{\text {in }}$; hydraulic diameter $d_{\text {hyd }}$ and heated length $l_{\text {heat }}$ of the channel; coefficients of local resistances at the inlet $\zeta_{\text {in }}$ and outlet $\zeta_{\text {out }}$ of the channel. The flow stability also depends on the orientation of the channel in the gravity field and on the direction of the flow with respect to the vector of this force.

One of the modern approaches to determining the areas of design and operating parameters at which it is possible to guarantee the absence of an unstable coolant flow in relation to the operating conditions of supercritical water reactors is to select generalized dimensionless parameters that determine the boundaries of the stable flow regions of supercritical media in channels (Ambrosini and Sharabi 2008):

$$
\begin{gathered}
N_{1}=\left(\beta_{\text {heat }} / c_{\text {exp, heat }}\right) \times\left(h_{\text {heat }}-h_{\mathrm{in}}\right), \\
N_{2}=\left(\beta_{\text {heat }} / c_{\text {exp,heat }}\right) \times(Q / G),
\end{gathered}
$$

where $Q$ is the power supplied to the coolant in the channel; $G$ is the fluid flow rate in the channel; $\beta_{\text {heat }}$ and $c_{\text {exp heat }}$ are the coefficient of volumetric expansion and specific 
heat at the pseudo-critical point. These parameters, as such, are analogs of dimensionless complexes that have already been used to describe the boundaries of stability on the map of flow regimes of two-phase flows (Saha and Zuber 1978). The first parameter $\left(N_{1}\right)$ can be considered as a value characterizing the fluid subcooling at the channel inlet to pseudocritical temperature, while the second parameter $\left(N_{2}\right)$ determines the change in the supercritical medium flow state as a result of heat supply to it. An example of a map with coordinates $N_{1}, N_{2}$ for fluid flow regimes at supercritical pressures is shown in Fig. 1 (Ambrosini et al. 2011).

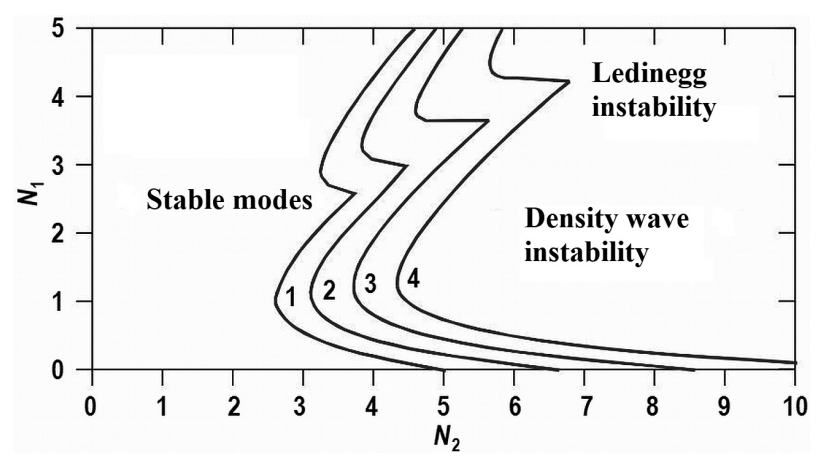

Figure 1. Map of flow regimes of a medium of supercritical parameters in a round pipe (coefficient of local resistance at the inlet $\zeta_{\text {in }}=20$; coefficient of local resistance at the outlet $\zeta_{\text {out }}: 1-20$; $2-10 ; 3-5 ; 4-2)$.

The threshold values of the defining parameters, shown in the figure by lines $1-4$, were obtained as a result of calculations according to the linearized model carried out for water ( $p=25 \mathrm{MPa})$, as well as for other media, i.e., carbon dioxide (8.0 MPa), freon-23 (5.7 MPa) and ammonia (15.0 MPa). The calculations (Ambrosini et al. 2011) were carried out for a channel in the form of a uniformly heated round pipe of vertical or horizontal orientation. The pipe internal pipe diameter $=8.36 \mathrm{~mm}$, length $=4.27 \mathrm{~m}$, wall roughness $=25 \mu \mathrm{m}$. The local resistance coefficient at the pipe inlet was constant; its value was taken to be 20 . The local resistance coefficient at the pipe outlet varied from 20 to 2 .

The suitability of the parameters $N_{1}$ and $N_{2}$ was checked for a general description of the stability of the system. Using the RELAP5 program code in (Ambrosini et al. 2011), calculations were made of thermal-hydraulic processes in a channel with supercritical water at a pressure of $25 \mathrm{MPa}$ (inlet temperature $280^{\circ} \mathrm{C}$, coolant flow rate $0.055 \mathrm{~kg} / \mathrm{s}$ and linear heat release rate $25 \mathrm{~kW} / \mathrm{m}$ ) simulating the situation at a peak power in the reactor. As the onset of oscillations was detected, the corresponding values of the dimensionless parameters $N_{1}$ and $N_{2}$ were recorded. As a result, the considered conditions in the channel were determined as stable and located far enough from the boundaries of the unstable region.

The paper (Gómez et al. 2008) presents an analysis of the thermal-hydraulic stability of a uniformly heated channel at supercritical water pressure by frequency and time methods based on a one-dimensional model. With the parameters of the coolant chosen in the projected SCWRs, the Ledinegg instability and pressure drop oscillations were practically excluded; therefore, the conditions were investigated when density waves arise in a supercritical aqueous medium. Drawing an analogy between the flows of a medium of sub- and supercritical pressure, as a result, for constructing maps of stability of the supercritical pressure water flow regimes, the authors of (Gómez et al. 2008) proposed two dimensionless complexes:

$$
\begin{gathered}
N_{1}{ }^{\prime}=\left[\left(v_{\text {out }}-v_{\text {in }}\right) / v_{\text {in }}\right]\left(L_{0} / L_{\mathrm{h}}\right), \\
N_{2}^{\prime}=\left(v_{\text {out }}-v_{\text {in }}\right) / v_{\text {in }},
\end{gathered}
$$

the physical meaning of which is similar to that considered earlier for $N_{1}$ and $N_{2}$. In this case, the change in the state of the supercritical medium due to heating in a channel of length $L_{\mathrm{h}}$ (dimensionless number $N_{2}{ }^{\prime}$, formula (4)) is expressed through the difference in specific volumes $v$ of the fluid at the inlet and outlet with respect to the value of the same parameter at the inlet to the channel, and the dimensionless supercritical water 'subcooling' (number $N_{1}^{\prime}$, formula (3)) is calculated as the fraction $L_{0} /$ $L_{\mathrm{h}}$ of number $N_{2}{ }^{\prime}$, where $L_{0}$ is the distance from the inlet at which the temperature value $T_{0}$, is reached, which determines the beginning of the 'pseudophase transition'. In calculations at a water pressure of $25 \mathrm{MPa}, T_{0}=350{ }^{\circ} \mathrm{C}$. is conventionally assumed in this work. In the case of uniform water heating along the length of the channel, the ratio $L_{0} / L_{\mathrm{h}}$ can be replaced by $\left(h_{0}-h_{\text {in }}\right) /\left(h_{\text {out }}-h_{\text {in }}\right)$, where $h_{0}$ is the single-valued function of temperature $T_{0}$ at a given pressure.

Work (Zhao et al. 2007) is devoted to the study of the thermal-hydraulic stability of the US SCWR by the frequency method in the approximation of a single-channel model. It is shown that it is possible to ensure the stable reactor operation by installing choke devices with the required values of the local resistance coefficients at the fuel assembly inlet. The heat capacity of the fuel allows softens the oscillations in the coolant flow rate. Heating the water elements of the moderator increases the stability of the coolant upward flow rate as a result of the fact that part of the heat from the fuel is transferred to the water elements and the water temperature at the inlet to the core increases when the flows are mixed. Thus, with the chosen flow pattern, the heating of the water elements leads to a decrease in the average density of the coolant and an increase in its average velocity, which ensures a reduction in the time the coolant moves through the channels. Increasing the moderator flow rate improves stability. The performed calculations revealed a higher sensitivity of the SCWR to changes in operating parameters than that of the BWR. According to the authors of (Zhao et al. 2007), this indicates the inadequacy of the classical stability analysis method using the criterion $D R$ (decay ratio) adopted abroad, the quantitative characteristic of which is the ratio of the amplitudes of successive oscillations of the system parameters (the 
less $D R$, the faster the oscillations damp). However, even a sufficiently small $D R$ value does not fully reflect the stability margin for a specific SCWR design. It is assumed that the degree of stability can be assessed by determining the sensitivity of the $D R$ criterion to changes in the operating conditions of a nuclear power plant.

The paper (Yi et al. 2004a) describes a study of the thermal-hydraulic stability of the SCLWR-H using linear analysis by the frequency method. Despite the low value of the coolant flow rate and the large change in its density in the SCLWR-H core, its thermal-hydraulic stability can be maintained both under the nominal operating mode and under the conditions of starting the reactor at constant supercritical pressure. This is possible due to an increase in the local resistance coefficient at the inlet to the fuel assembly. It is noted that the boundaries of the stability region can also be expanded by decreasing the ratio of the reactor power to the coolant flow rate or by decreasing the temperature at the inlet to the core, while the values of the $D R$ criterion will decrease (Fig. 2).

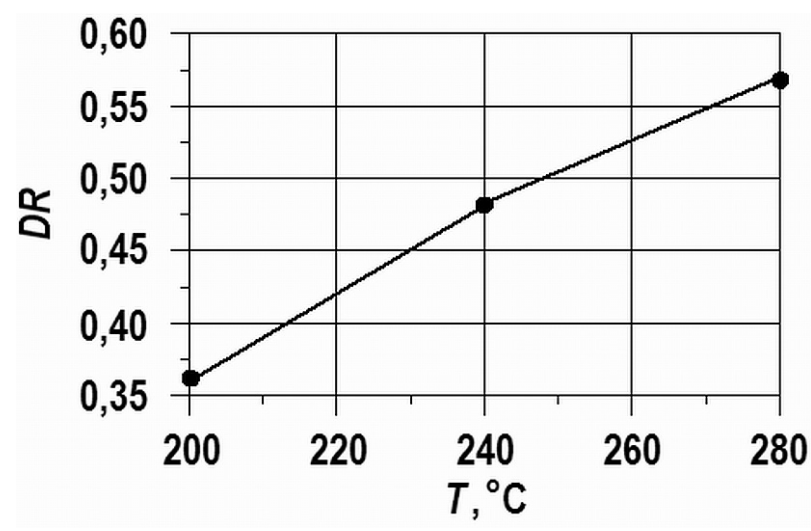

Figure 2. Influence of water temperature at the inlet to the core on the thermal-hydraulic stability (Yi et al. 2004a).

The studies (Liu et al. 2014, Hou et al. 2011) were carried out for the thermal and fast regions of the reactor core with a mixed neutron spectrum, namely SCWR-M. A linear analysis of the thermal-hydraulic stability with respect to density wave disturbances by frequency and time methods was carried out and the stability boundaries were established.

The results of the frequency analysis method showed that the coolant flow rate in the thermal region is stable in a wide range of operating parameters. An increase in the mass flow rate of the coolant has a positive effect on the stability of the system. The importance of the second natural frequencies of the system was identified. This led to the need for a nonlinear stability analysis. The time analysis method showed that the oscillations in the mass flow rate of water at the inlet and outlet of each channel do not coincide in phase. Apparently, this was due to the use of a fixed boundary value for the flow rate at the inlet to the channels.

The stability of the SCWR-M fast-spectrum core zone was determined in a wide range of operating conditions.
The stability of the parallel channel system is determined by the hottest channel, the parameters of which are most susceptible to oscillations in unstable modes. The higher the specific power of the hottest channel, the more unstable the system is. An increase in the mass flow rate of the coolant has a positive effect on stability. Systems with a uniform axial power distribution are less stable than systems with a cosine or forked distribution.

The results of the analysis by two methods in both works are in good agreement in assessing the limiting stability of the system.

The authors of (Xiong et al. 2013) described the results of checking the previously obtained experimental data on the thermal-hydraulic stability of parallel channels. The analysis was carried out using a one-dimensional program code, which implements a temporary method for studying stability. A comparison was made of three different models of the geometry of the working area. The calculated and experimental data obtained are in good agreement with each other, which allows us to speak about the admissibility of using a one-dimensional code to determine the flow stability boundaries in parallel channels of a supercritical pressure medium. The study of the influence of geometry showed the inadmissibility of excluding the inlet and outlet sections in the numerical analysis of the coolant flow rate stability. A relatively simple geometric model for generalized numerical stability analysis was proposed. It is noted that the system stability is negatively affected by the high temperature of the coolant at the inlet to the working section and the high mass flow rate of the heat exchange medium.

In (Tian et al. 2012), a study of the thermal-hydraulic stability of the CSR1000 reactor by the frequency analysis method using a one-dimensional numerical model is presented.

It was found that the range of parameters in which the thermal-hydraulic instability is observed is directly determined by the point at which the coolant reaches the pseudocritical temperature. In addition, the boundary of the stability region of the CSR1000 strongly depends on the value of the hydraulic resistance coefficient adopted in the calculations. It is noted that, depending on the operating parameters in the reactor, both the Ledinegg instability and the density wave instability can occur.

In (Yagov et al. 2011), computational studies of the aperiodic and periodic stability of the coolant flow in the fuel assemblies of the VVER-SCP reactor are presented. The authors carried out a sensitivity analysis of the reactor stability to the mass flow rate of water as well as to the hydraulic resistance coefficients of the spacer grids, the lower support grid and the fuel assembly head. The calculation results showed no multivaluedness of the hydrodynamic characteristics of fuel assemblies; therefore, there is no aperiodic instability of the coolant flow. To ensure the periodic stability of the coolant flow, it is necessary to use throttling devices with a coefficient of local hydraulic resistance greater than 12 .

The work (Chistov et al. 2015) is focused on determining the instability region of the coolant flow rate in 
the first circulation circuit of a two-circuit NPP and in the second circuit of a steam generator (SG) for the nominal operating mode of the SCPS-600 reactor plant depending on the key parameters, as well as the region of flow stability in isolated parallel channels of the reactor core, taking into account the non-uniformity of heat release for different groups of fuel assemblies. The frequency and time analysis methods were used for a one-dimensional homogeneous model of the coolant flow. It was shown that the stability of the coolant flow rate in both circuits at the nominal mode of the SCPS-600 was provided more than enough. This fact was confirmed by calculations of transient processes.

In (Su et al. 2013), the thermal-hydraulic instability of the flow in two parallel channels with supercritical pressure water was investigated by the time analysis method. The coolant flow rate instability was studied using the small-disturbances method. The ultimate stability boundary was obtained, and the sensitivity of thermal-hydraulic stability to various parameters, such as mass flow rate of the coolant, heat flow, water temperature at the entrance to the channels and pressure in the system, was determined.

It is shown that stability is greatly influenced by the pressure drop, the change in the value and distribution of the mass flow rate of the coolant between the heated channels. The stability of the parallel channels is mainly determined by the pressure drop. With an increase in the mass flow rate and pressure in the system, as well as with a decrease in the heat flow, the stability of the flow rate in the parallel channels increases. A dimensionless analysis of the boundaries of the thermal-hydraulic stability indicated an analogy between the key criteria in systems with water of sub- and supercritical pressure. The influence of the temperature at the inlet is ambiguous at low and high values of water 'pseudo-subcooling'. Small changes in the coolant density and in the pressure drop cause instability in the parallel channels. The authors note the importance of taking into account the lag effects and feedbacks between the mass flow rate, the density of the medium and the pressure drop.

In (Dutta et al. 2015a, 2015b), a study of the thermal-hydraulic stability of a supercritical water reactor of the CANDU-type is presented. The studies were carried out by the temporary analysis method in two versions: on the basis of a single-channel model and a model of a system of parallel channels. The conditions for the occurrence of oscillations in the form of 'density waves' were determined. The authors studied the influence of various parameters on the reactor stability, such as the coolant mass flow rate, the operating pressure, the profile of the axial heat flux, the local resistance coefficient and the friction coefficient.

It is argued that predicting the flow stability boundaries using a single-channel model may be inaccurate due to the fact that this model does not take into account the radial change in the power of heat release in the reactor and the possibility of instability in the form of oscillations of the coolant flow in the parallel channels in antiphase.

\section{Neutronic thermal-hydraulic stability}

The analysis of neutronic thermal-hydraulic stability takes into account that one or another type of instability of the coolant flow in a particular reactor facility should always be considered as a complex phenomenon, in which various processes (hydrodynamic, thermal or neutronic) are simultaneously involved, occurring under certain boundary conditions and closely related with the features of the scheme and design of this facility.

In (Yi et al. 2004b), a mathematical model was developed based on the equations of thermal hydraulics and point neutron kinetics as well as the temperature dynamics of fuel elements to study the neutronic thermal-hydraulic stability of the SCLWR-H. The authors considered the reactor operation at rated power, at partial loads, as well as at power rise during the reactor startup under conditions of constant supercritical pressure.

It is noted that the neutronic effects have a significant impact on the reactor stability. This illustrates the comparison of the parameters of the oscillations of the $D R$ system, calculated using the thermal-hydraulic and neutronic thermal-hydraulic models (see Figs 2, 3).

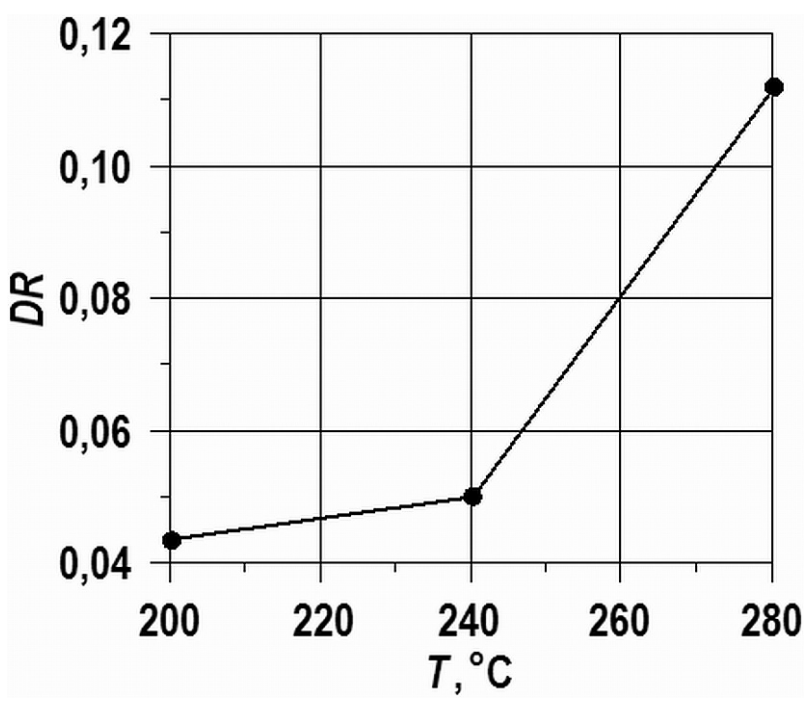

Figure 3. Influence of water temperature at the inlet to the core on the neutronic thermal-hydraulic stability (Yi et al. 2004b).

It was found that, on the whole, the SCLWR-H design meets the requirements for the neutronic thermal-hydraulic stability when the reactor is operating at its rated power. Instability can occur at low loads. At the same time, a negative influence is exerted by the effect of feedback with the moderator density. An increase in the density coefficient of reactivity decreases stability. Due to the long delay of heat transfer to water in the moderator rods, the joint consideration of the interacting neutronic and thermal-hydraulic characteristics acquires particular importance in the analysis of the stability of this reactor. 
The results of the study of the thermal-hydraulic and neutronic thermal-hydraulic stability of the US SCWR, obtained using the frequency analysis method to a one-dimensional numerical model, are presented in (Buongiorno and MacDonald 2003, Zhao et al. 2008).

The calculations of instabilities, when oscillations in operating parameters coincide in phase within the entire core, showed that, under normal conditions, such oscillations quickly damp. The rate of this process is characterized by the obtained $D R$ values, which in cases of the thermal-hydraulic and neutronic thermal-hydraulic instability are equal to 0.20 and 0.007 , respectively, which is significantly lower than the limit values usually taken when the BWR stability is assessed $(0.5$ and 0.25 ). The sensitivity analysis showed that an increase in the coefficient of local resistances at the inlet sections increases the system stability, and an increase in the feedback coefficient for the coolant density decreases it. Compared with the axial profile of the cosine-shaped heat release power, the uniform profile increases stability, while the asymmetric profile sloping downwards decreases it. It is shown that this reactor is characterized by a weak connection between neutron kinetics and thermal hydraulics. In this case, the main moderators of neutrons are water rods, in which oscillations in the water density are insignificant; in addition, the high subcriticality of the first subcritical regime, compared to that of the BWR, provides fast damping of neutron oscillations.

In (Cervi and Cammi 2018), a study of the thermal-hydraulic and neutronic thermal-hydraulic stability of the SCWR concept, carried out by the frequency analysis method, is described. The stability of this reactor plant in the nominal operating range of parameters was revealed, and a high sensitivity of the stability of the system to the coolant mass flow rate during transient processes was found.

\section{Summarizing comments}

Below is a summary table, where, in addition to the purpose and main characteristics of the computational models, additional information is provided regarding the use of ratios to determine the coefficients of friction and heat transfer on the channel walls in the analysis of the thermal-hydraulic stability.

Based on the data in the table, we can note the following: of all the works, only two (Hou et al. 2011, Chistov et al. 2015) considered the features of the thermal-hydraulic stability of reactors, in which the coolant temperature at the inlet to the core exceeded the pseudocritical value at a given pressure.

To determine the boundaries of the supercritical coolant flow stability, computational schemes of varying degrees of complexity were used, including three-dimensional thermal-hydraulic and complex neutronic thermal-hydraulic models. It is shown that the preliminary generalized stability analysis can be performed by the frequency or time analysis methods using one-dimensional one-channel models.

In the case of one-dimensional one-channel models, the heat flux density on the channel walls is usually a given value, constant or a function that varies along the channel length. Thus, the hydrodynamic connection between adjacent channels, combined by the inlet and outlet collectors in the core, is not taken into account, and the thermal interaction of the channel under consideration with its environment is also disregarded. In this respect, models that include two or more parallel channels have an undoubted advantage.

Table 1. Purpose and main characteristics of computational models in works (Gómez et al. 2008, Zhao et al. 2007, Yi et al. 2004a, Liu et al. 2014, Hou et al. 2011, Xiong et al. 2013, Tian et al. 2012, Yagov et al. 2011, Chistov et al. 2015, Su et al. 2013, Dutta et al. 2015a, Dutta et al. 2015b, Yi et al. 2004b, Buongiorno and MacDonald 2003, Zhao et al. 2008, Cervi and Cammi 2018)

\begin{tabular}{|c|c|c|c|c|c|}
\hline Work & $\begin{array}{c}\text { Simulated reactor } p, \mathrm{MPa} ; \\
T_{\text {in/out }},{ }^{\circ} \mathrm{C}\end{array}$ & $\begin{array}{c}\text { Thermal- } \\
\text { hydraulic model }^{1)}\end{array}$ & $\begin{array}{l}\text { Ratios for the } \\
\text { friction coeff. }^{2)}\end{array}$ & $\begin{array}{c}\text { Ratios for the } \\
\text { heat-trans. coeff. }\end{array}$ & $\begin{array}{c}\text { Neutronic } \\
\text { model }\end{array}$ \\
\hline (Gómez et al. 2008) & SCWR 25; 280/500 & $\mathrm{S}$ & $\mathrm{Ha}$ & - & - \\
\hline (Zhao et al. 2007) & US SCWR 25; 280/500 & $\mathrm{S}$ & - & D-B & - \\
\hline (Yi et al. 2004a) & SCLWR-H 25; 280/500 & $\mathrm{S}$ & $\mathrm{B} 1$ & $\mathrm{~K}-\mathrm{O}$ & - \\
\hline (Liu et al. 2014) & SCWR-M 25; 280/407.7 & $\mathrm{PCh}$ & - & - & - \\
\hline (Hou et al. 2011) & SCWR-M 25; 407.7/510 & $\mathrm{PCh}$ & Bl-McA & - & - \\
\hline (Xiong et al. 2013) & SCWR 25; 220/450 & $\mathrm{PCh}$ & $\mathrm{Ha}$ & - & - \\
\hline (Tian et al. 2012) & CSR1000 25; 280/500 & $\mathrm{S}$ & $\mathrm{Ha}, \mathrm{Bl}, \mathrm{F}$ & $\mathrm{K}-\mathrm{O}$ & - \\
\hline (Yagov et al. 2011) & VVER-SCP $24.5 ; 290 / 540$ & $\mathrm{~S}$ & - & - & - \\
\hline (Chistov et al. 2015) & SCPS-600 24.5; 390/500 & $\mathrm{S}$ & - & - & - \\
\hline (Su et al. 2013) & SCWR 25; 280/500 & $\mathrm{PCh}$ & Bl-McA & - & - \\
\hline (Dutta et al. 2015a) & CANDU SCWR $25 ; 350 / 625$ & $\mathrm{~S}$ & Ch-Ch, C-W, F & - & - \\
\hline (Dutta et al. 2015b) & CANDU SCWR $25 ; 350 / 625$ & $\mathrm{PCh}$ & $\mathrm{Ch}-\mathrm{Ch}$ & - & - \\
\hline (Yi et al. 2004b) & SCLWR-H 25; 280/500 & $\mathrm{S}$ & $\mathrm{B} 1$ & $\mathrm{~K}-\mathrm{O}$ & Point, 6 groups \\
\hline (Buongiorno and MacDonald 2003) & US SCWR $25 ; 280 / 500$ & $\mathrm{~S}$ & - & - & Point \\
\hline (Zhao et al. 2008) & US SCWR $25 ; 280 / 500$ & $\mathrm{~S}$ & - & - & Point \\
\hline (Cervi and Cammi 2018) & SCWR $25 ; 280 / 500$ & $\mathrm{~S}$ & $\mathrm{~F}, \mathrm{P}$ & M & Point, 6 groups \\
\hline
\end{tabular}

Notes: ${ }^{1)} \mathrm{S}=$ single-channel; $\mathrm{PCh}$ - parallel channels;

${ }^{2)} \mathrm{Ha}$ - Haaland; Bl - Blasius; F - Filonenko; McA - McAdams; Ch-Ch - Churchill-Chu; C-W - Colebrook-White; P - Popov;

3) D-B - Dittus-Boelter; K-O - Koshizuka-Oka; M - Mokry. 
Particular consideration should be given to the development of one-dimensional models, in which the heat transfer between the coolant moving in the cells between the heat-generating and other elements of the core structure in non-stationary (transient) modes, when the heat capacity of materials can play a significant role.

In thermal SCWRs, where the so-called 'water rods' serve as the moderator of neutrons, taking into account the heat transfer between these rods and the coolant is especially important, since a change in the water rod temperature, and hence the moderator density, can lead to the neutronic thermal-hydraulic instability.

In any case, the frictional resistance and heat transfer in channels with supercritical water parameters should be taken into account using relations specially developed for the case of a significant change in the thermal-physical properties of the coolant with temperature and pressure near the critical point.

It is important to keep in mind that at supercritical pressure in the vicinity of the 'pseudophase transition', the regularities of friction resistance can have peculiarities, and the heat transfer by its nature can vary greatly, i.e., depending on conditions it can be normal, improved or deteriorated. The ratios for the coefficients of friction and heat transfer should be tested for use in the calculations of such complex structures as fuel assemblies for nuclear reactors. Unfortunately, the above considerations, as can be seen from Tab. 1 are not observed in the listed one-dimensional models in most cases.

As for three-dimensional models, in which a detailed calculation of flow characteristics is carried out using modern CFD codes, it should be noted that the methods for determining the coefficients of turbulent transfer of momentum and heat in supercritical media have not yet been sufficiently developed. The results obtained in this way are not entirely reliable and, therefore, do not have any special advantages over the data found by one-dimensional models, in which the parameters averaged over the channel cross-section and empirical coefficients of resistance and heat transfer are used.

Undoubtedly, to obtain the recommendations necessary to substantiate the stable operation and safety of any nuclear power plant, one should use complex neutronic thermal-hydraulic computational models that fully take into account the feedbacks between the reactor reactivity, pressure drop, flow rate, and coolant temperature.

\section{Conclusion}

The main feature of the stability of the coolant flow in the core of supercritical water reactors is associated with the strong dependence of the physical properties of water on temperature and pressure in the region of the 'pseudo-phase transition' near the critical point.

By tradition and by analogy with a boiling coolant, in computational studies of the thermal-hydraulic stability of systems with supercritical water, frequency and time analysis methods are used with process models of different levels of complexity. One-dimensional non-stationary models with one or two channels combined by inlet and outlet collectors have received the greatest development. Based on the results of calculations using these models, a number of useful parametric and generalized dependencies have been obtained that determine the boundaries of the existence of stable flow and heat removal regimes.

Further improvement of the computational models involves the inclusion of the hydrodynamic and thermal interaction of the channel (cell) under consideration with the coolant with the surrounding elements of the core (adjacent channels, fuel rods, moderator water rods, etc.) into the non-stationary process under study. In this case, as the closing relations for the frictional resistance and heat transfer, universal dependences should be mainly used that take into account both the variability of the properties of water and the possibility of changing the flow and heat transfer regimes. These dependencies should be tested for use in calculations of structures that are as close as possible to real fuel assemblies of nuclear reactors.

The final conclusions about the reliability and safety of a nuclear power plant in nominal and transient modes should be based on the results of calculations performed using complex models of neutronic thermal-hydraulic stability, built on the basis of modern advances in neutron physics and thermal physics.

\section{References}

- Ambrosini W (2007) On the Analogies in the Dynamic Behaviour of Heated Channels with Boiling and Super-Critical Fluids. Nuclear Engineering and Design 237: 1164-1174. https://doi.org/10.1016/j. nucengdes.2007.01.006

- Ambrosini W, Bilbao y León S, Yamada K (2011) Results of the IAEA Benchmark Exercise on Flow Stability in Heated Channels with Supercritical Fluids. The $5^{\text {th }}$ International Symposium on Supercritical Water-Cooled Reactors (ISSCWR-5). March 13-16, Vancouver, Canada: 115.

- Ambrosini W, Sharabi M (2008) Dimensionless Parameters in Stability Analysis of Heated Channels with Fluids at Supercritical
Pressures. Nuclear Engineering and Design 238: 1917-1929. https:// doi.org/10.1016/j.nucengdes.2007.09.008

- Bergles AE (1977) Review of Instabilities in Two-Phase Systems. Two-Phase Flows and Heat Transfer, 1977, v. 1, 383-422.

- Buongiorno J, MacDonald PE (2003) Supercritical Water Reactor (SCWR). Progress Report for the FY-03 Generation-IV. R\&D Activities for the Development of the SCWR in the U.S. Report INEEL/ EXT03-03-01210. Idaho National Engineering and Environmental Laboratory, USA.

- Cervi E, Cammi A (2018) Stability Analysis of the Supercritical Water Reactor by Means of the Root Locus Criterion. Nuclear 
Engineering and Design 338: 137-157. https://doi.org/10.1016/j. nucengdes.2018.08.004

- Chistov AS, Savikhin OG, Ovchinnikov VF, Nikolaev MY (2015) Investigation of the Stability of the Coolant Flow Rate in a Two-Circuit NPP with a VVER-SCP Reactor. Strength and Plasticity Problems 77(3): 309-318. [in Russian] https://doi.org/10.32326/18149146-2015-77-3-309-318

- Dutta G, Zhang C, Jiang J (2015a) Analysis of Flow Induced Density Wave Oscillations in the CANDU Su-Percritical Water Reactor. Nuclear Engineering and Design 286: 150-162. https://doi. org/10.1016/j.nucengdes.2015.02.007

- Dutta G, Zhang C, Jiang J (2015b) Analysis of Parallel Channel Instabilities in the CANDU Supercritical Water Reactor. Annals of Nuclear Energy 83: 264-273. https://doi.org/10.1016/j.anucene.2015.04.023

- Gómez TO, Class A, Lahey RT, Schulenberg T (2008) Stability Analysis of a Uniformly Heated Channel with Supercritical Water. Nuclear Engineering and Design 238: 1930-1939. https://doi org/10.1016/j.nucengdes.2007.10.031

- Hou D, Lin M, Liu P, Yang Y (2011) Stability Analysis of Parallel-Channel Systems with Forced Flows under Supercritical Pressure. Annals of Nuclear Energy 38: 2386-2396. https://doi.org/10.1016/j. anucene.2011.07.021

- Liu P, Hou D, Lin M, Kuang B, Yang Y (2014) Stability Analysis of Parallel-Channel Systems under Super-Critical Pressure with Heat Exchanging. Annals of Nuclear Energy 69: 267-277. https://doi. org/10.1016/j.anucene.2014.02.021

- Saha P, Zuber N (1978) An Analytical Study of the Thermally Induced Two-Phase Flow Instabilities Including the Effect of Thermal Non-Equilibrium. International Journal of Heat and Mass Transfer 21: 415-426. https://doi.org/10.1016/0017-9310(78)90075-3

- Su Y, Feng J, Zhao H, Tian W, Su G, Qiu S (2013) Theoretical Study on the Flow Instability of Supercritical Water in the Parallel Channels. Progress in Nuclear Energy 68: 169-176. https://doi. org/10.1016/j.pnucene.2013.06.005
- Tian X, Tian W, Zhu D, Qiu S, Su G, Xia B (2012) Flow Instability Analysis of Supercritical Water-Cooled Reactor CSR1000 Based on Frequency Domain. Annals of Nuclear Energy 49: 70-80. https:/ doi.org/10.1016/j.anucene.2012.06.023

- Xiong T, Yan X, Huang S, Yu J, Huang Y (2013) Modeling and Analysis of Supercritical Flow Instability in Parallel Channels. International Journal of Heat and Mass Transfer 57: 549-557. https://doi. org/10.1016/j.ijheatmasstransfer.2012.08.046

- Yadigaroglu G (1984) Instability of Two-Phase Flow and Propagation of Disturbances. In: Delhaye JM, Giot M, Rietmuller ML (Eds) Thermohydraulics of Two-Phase Systems for Industrial Design and Nuclear Engineering. Moscow. Energoatomizdat Publ., ch. 17: 271307. [in Russian]

- Yagov PV, Churkin AN, Mokhova OV (2011) Analysis of the Thermohydrodynamic Stability of the Coolant Flow and Uneven Heating in VVER-SCP Fuel Assemblies. VANT. Series: NPP Safety Ensurance 29: 82-91. [in Russian]

- Yi TT, Koshizuka S, Oka Y (2004a) A Linear Stability Analysis of Supercritical Water Reactors, (I) Thermal-Hydraulic Stability. Journal of Nuclear Science and Technology 41: 1166-1175. https://doi.or g/10.1080/18811248.2004.9726345

- Yi TT, Koshizuka S, Oka Y (2004b) A Linear Stability Analysis of Supercritical Water Reactors, (II) Coupled Neutronic Thermal-Hydraulic Stability. Journal of Nuclear Science and Technology 41: 1176-1186. https://doi.org/10.1080/18811248.2004.9726 346

- Zhao J, Saha P, Kazimi MS (2007) Hot-Channel Stability of Supercritical Water-Cooled Reactors-II: Effect of Water Rod Heating and Comparison with BWR Stability. Nuclear Technology 158: 174 190. https://doi.org/10.13182/NT07-A3834

- Zhao J, Saha P, Kazimi MS (2008) Coupled Neutronic and Thermal-Hydraulic Out-of-Phase Stability of Supercritical Water-Cooled Reactors. Nuclear Technology 164: 20-33. https://doi.org/10.13182/ NT08-A4005 